\title{
Nanoclustering as a dominant feature of plasma membrane organization
}

\author{
Maria F. Garcia-Parajo ${ }^{1,2, \uparrow}$, Alessandra Cambi ${ }^{3,4, *}$, Juan A. Torreno-Pina ${ }^{1}$, Nancy Thompson ${ }^{5}$ and \\ Ken Jacobson ${ }^{6,7,9}$
}

\begin{abstract}
Early studies have revealed that some mammalian plasma membrane proteins exist in small nanoclusters. The advent of super-resolution microscopy has corroborated and extended this picture, and led to the suggestion that many, if not most, membrane proteins are clustered at the plasma membrane at nanoscale lengths. In this Commentary, we present selected examples of glycosylphosphatidyl-anchored proteins, Ras family members and several immune receptors that provide evidence for nanoclustering. We advocate the view that nanoclustering is an important part of the hierarchical organization of proteins in the plasma membrane. According to this emerging picture, nanoclusters can be organized on the mesoscale to form microdomains that are capable of supporting cell adhesion, pathogen binding and immune cell-cell recognition amongst other functions. Yet, a number of outstanding issues concerning nanoclusters remain open, including the details of their molecular composition, biogenesis, size, stability, function and regulation. Notions about these details are put forth and suggestions are made about nanocluster function and why this general feature of protein nanoclustering appears to be so prevalent.
\end{abstract}

KEY WORDS: Plasma membrane, Protein nanoclustering, Super-resolution microscopy

\section{Introduction}

Organization by compartmentalization is a general property of natural systems that efficiently facilitates and orchestrates biological events in space and time. Cells are primary examples of well-defined biological compartments within tissues. However, cells also exhibit a number of compartmentalization strategies, including membrane-delimited intracellular organelles (e.g. endosomes and lysosomes) and multi-enzyme complexes. This compartmentalization of specific cellular functions, through spatial localization, increases regulation efficiency. In addition,

${ }^{1}$ ICFO-Institut de Ciencies Fotoniques, Mediterranean Technology Park, 08860 Castelldefels (Barcelona), Spain. ${ }^{2}$ ICREA-Institució Catalana de Recerca i Estudis Avançats, 08010 Barcelona, Spain. ${ }^{3}$ Nanobiophysics, MIRA Institute for Biomedical Technology and Technical Medicine and MESA+ Institute for Nanotechnology, University of Twente, 7522 NB Enschede, The Netherlands. ${ }^{4}$ Department of Tumor Immunology, Radboud Institute for Molecular Life Sciences, Radboud University Medical Center, 6500 HB Nijmegen, The Netherlands. ${ }^{5}$ Department of Chemistry, University of North Carolina at Chapel Hill, Chapel Hill, NC 27599-3290, USA.

${ }^{6}$ Department of Cell Biology and Physiology, University of North Carolina at Chapel Hill, Chapel Hill, NC 27599-7090, USA. 'Tineberger Comprehensive Cancer Center, University of North Carolina at Chapel Hill, Chapel Hill, NC 27599, USA.

*Present address: Department of Tumor Immunology, Radboud Institute for

Molecular Life Sciences, Radboud University Medical Center, 6500 HB Nijmegen, The Netherlands.

•Authors for correspondence (maria.garcia-parajo@icfo.es; frap@med.unc.edu) the plasma membrane with its extracellular matrix and the subjacent membrane-associated cytoskeleton is also highly organized - with areas in which specific components are enriched or to which they are recruited - thereby providing specialized cellular regions, such as the basal and the apical membrane in polarized cells, the immunological synapse in interacting leukocytes or multimolecular membrane-cytoskeleton assemblies that include focal adhesions and podosomes. Once considered a relatively unstructured 'sea' of lipids and proteins that are potentially able to form aggregates (Singer and Nicolson, 1972), the plasma membrane is now widely accepted as being highly compartmentalized, thus allowing lipids and proteins to be organized in specific regions of varying size and composition (Kusumi et al., 2011; Maxfield, 2002; Nicolson, 2013).

One of the most prominent concepts for membrane compartmentalization refers to the 'lipid raft hypothesis'. The raft model originated from a study more than 25 years ago (van Meer et al., 1987) and has been extensively reviewed since then (Lingwood and Simons, 2010; Simons and Toomre, 2000). The lipid raft notion proposes the existence of small plasma membrane compartments that are enriched in cholesterol and sphingolipids, and populated by 'raftophilic' proteins such as, for example, glycosylphosphatidyl-anchored proteins (GPI-APs). Persistent controversies have led to a provisional contemporary definition that emphasizes the small and transient nature of putative lipid rafts (Pike, 2006). Yet, when considering the current, general view about the nature of lipid rafts, it must be borne in mind that membranes are rich in proteins (Jacobson et al., 2007) and that any preferential residence of membrane proteins in regions populated by raft lipids most likely occurs only on a very small spatial and temporal scale (Eggeling et al., 2009). At present, the existence of relatively large, and predominantly 'lipid raft' domains is expected to be the exception rather than the rule.

Another prominent concept for plasma membrane compartmentalization is based on the meshwork of filamentous proteins including F-actin and spectrin that immediately underlie the plasma membrane. This model originated over three decades ago on the basis of lateral diffusion studies of proteins in the membranes of red blood cells (Sheetz, 1983) and was later expanded by work from Akihiro Kusumi's group, who termed it the membrane cytoskeleton 'fence' (Kusumi et al., 2005). They also coined the term 'anchored transmembrane picket', which refers to the more or less regular array of transmembrane proteins (posts) that are anchored to the underlying membrane-associated cytoskeletal fence serving to compartmentalize the membrane. Thus, long-range diffusion of both transmembrane and lipidanchored proteins is restricted because it is rate-limited by fluctuations in the cytoskeletal 'fence-picket' structure that 
permits the inter-compartmental barriers to be traversed by the diffusing molecule. By contrast, short-range diffusion within each compartment is much more rapid.

Other notions have also been put forth with regard to factors that govern plasma membrane compartmentalization on different length and time scales, such as lipid shells (Anderson and Jacobson, 2002), tetraspanin-enriched microdomains (Yáñez-Mó et al., 2009) and galectin lattices (Lajoie et al., 2009). Moreover, recent evidence suggests that these possible local organizers are not independent from each other but, rather, act in a synergistic manner that remains to be precisely defined (Delaguillaumie et al., 2004; Lajoie et al., 2009).

The term 'microdomain' is generally employed to describe membrane compartmentalization that occurs either naturally or is ligand induced. However, the size of such domains can vary from a few nanometers to several micrometers, most probably owing to their different molecular composition and different capacities to coalesce with or segregate from each other. A plausible hypothesis is that plasma membrane compartmentalization is based on a hierarchical organization of molecules that ranges from classic protein oligomerization to nanometer-sized or micrometer-sized clusters, which may provide multi-scale regulation of membrane protein function. However, in this Commentary, we will focus on organization at the smallest scale, namely, the association of membrane proteins into clusters of nanometer dimensions into which other proteins or lipids may be incorporated.

The rapid development of super-resolution optical microscopy (Table 1) and single-particle tracking approaches in the past few years has provided - and is continuing to provide - indisputable evidence for nanoclustering of many, if not most, proteins on mammalian cell plasma membranes - even in the absence of any activation or ligand binding. Whereas examples of nanoclustering in neurobiology can be found in a recent review (Willig and Barrantes, 2014), we will focus here on some other salient examples of receptor nanoclustering, including those involved in pathogen binding, cell adhesion and the immune response.

\section{The diversity of membrane protein nanoclustering Lipid-anchored proteins}

Some of the smallest documented nanoclusters are those that contain GPI-APs. These extracellular, lipid-linked proteins constitute a diverse set of proteins tethered to the outer leaflet of the plasma membrane that exhibits specific sorting and signaling properties. GPI-APs are regulated by alterations in cholesterol and sphingolipid levels in cell membranes and, as such, have been considered to be classic lipid-raft markers (Mayor and Riezman, 2004). The first studies discussing the unusual nature of GPI-AP nanoclustering were reported by two different groups using complementary approaches; i.e. chemical crosslinking (Friedrichson and Kurzchalia, 1998) and fluorescence resonance energy transfer (FRET) (Varma and Mayor, 1998). Later, using a combination of homo- and heteroFRET techniques, it was shown that GPI-APs co-exist on the cell membrane as mixtures of monomers together with a fraction $(20$ $40 \%)$ that includes cholesterol-sensitive oligomers $(<5 \mathrm{~nm})$ composed of at most four molecules; this was shown for a diverse set of GPI-AP species (Sharma et al., 2004). Surprisingly, the relationship between monomers and nanoclusters did not obey the mass-action law that would be expected for a classic chemical equilibrium, because the percentages of GPI-APs nanoclusters and their molecular density were concentration-independent (Mayor and Rao, 2004). Importantly, GPI-AP nanoclustering has been more recently confirmed by super-resolution microscopy techniques (Table 1), including near-field scanning optical microscopy (NSOM) (van Zanten et al., 2009) (Fig. 1) and photoactivated localization microscopy (PALM) (Sengupta et al., 2011). Single-particle tracking (SPT) and fluorescence correlation spectroscopy (FCS) studies on nanoscale volumes (Eggeling et al., 2009) have provided further evidence for the existence of small GPI-AP nanoclusters in living cell membranes - although, as recently pointed out, an entirely consistent picture has not yet emerged owing to the high number of different techniques employed (Klotzsch and Schütz, 2013).

Other lipid-tethered proteins at the inner leaflet of the membrane, e.g. the Ras family of GTPases, also form nanoclusters that are composed of small numbers of proteins, as observed by immunogold electron microscopy (EM) (Parton and Hancock, 2004). Ras isoforms are distinguished by differences in the structure of their lipid anchors; e.g. K-Ras is tethered to the membrane by a farnesyl group and a polybasic region, whereas $\mathrm{H}-\mathrm{R}$ as is anchored by two palmitoyl chains and a farnesyl group. EM imaging of the membrane-targeting regions of these two Ras isoforms has shown that fewer than ten copies of both K-Ras and $\mathrm{H}$-Ras are localized in largely separated nanoclusters of less than $30 \mathrm{~nm}$ within the inner leaflet of the plasma membrane (Plowman et al., 2005; Prior et al., 2003). Ras nanoclusters had lifetimes of less than 1 second, were immobile and constituted about $40 \%$ of the total Ras population, with the remaining population being monomeric and laterally mobile (Murakoshi et al., 2004). The properties of Ras nanoclusters are complicated by their dependence on the structure of the membrane anchor and the hyper-variable region of the protein moiety, the presence of cholesterol, extrinsic clustering factors and whether the isoform is loaded with GTP or GDP (Harding and Hancock, 2008). For example, when loaded with GDP but not GTP, H-Ras nanoclusters were localized in lipid rafts based on the affinity of their lipid chains for raft domains and by the fact that the localization required cholesterol (Harding and Hancock, 2008). By contrast, the K-Ras targeting domain also localized in nanodomains but this localization did not depend on cholesterol. In general, the field appears to be moving towards a consensus that GPI-AP and Ras nanoclusters constitute nucleation sites for signaling at the cell membrane that can grow and be stabilized by the recruitment of additional components to create active functional platforms for specific cellular functions (Cebecauer et al., 2010; Mayor and Rao, 2004; van Zanten et al., 2009).

Of considerable interest is the nature of the factors that regulate and stabilize nanoclusters of lipid-anchored proteins. In general, lipid-linked protein nanoclusters might be stabilized by cis associations of their protein ectodomains, cytosolic domains, lipid anchors or a combination of these. For example, Akihiro Kusumi's group recently showed that ectodomain interactions are the primary factor in transiently stabilizing homodimers of the GPI-AP CD59, with cholesterol and other lipids having secondary stabilizing roles (Suzuki et al., 2012). Furthermore, in supported lipid bilayers, Ras GTPases dimerize using a specific motif in their cytosolic domains (Lin et al., 2014). These studies suggest that protein-protein interactions among identical lipid-linked proteins play a significant role with respect to the stability of lipid-linked protein nanoclusters.

Accessory molecules that are located outside of the membrane might also be key to the formation, size and stability 
Table 1. Super-resolution microscopy approaches to image nanoclusters in membranes

\begin{tabular}{lll}
\hline Method & Description & Benefits \\
\hline $\begin{array}{l}\text { Single-molecule } \\
\text { localization } \\
\text { microscopy (SMLM) }\end{array}$ & $\begin{array}{l}\text { Localization of individual molecules that results in high-precision maps } \\
\text { of molecular positions. This group of methods is based on stochastic } \\
\text { activation of dispersed fluorophores and subsequent analysis to obtain lateral precision (10- } \\
\text { the centers of the fluorescent spots within the images. }\end{array}$ & $\begin{array}{l}50 \text { nm) spatial mapping of } \\
\text { molecular positions. }\end{array}$ \\
diffraction-limited \\
image
\end{tabular}

Main variants:

-PALM and fPALM, based on photoactivatable fluorescent probes -STORM, dSTORM and the BLINK technique, based on photoswitchable fluorescent probes

Stimulated emission microscopy depletion (STED)

Enhanced resolution results from narrowing of the point-spread function. It is based on a doughnut-shaped excitation beam superimposed on the primary Gaussian profile excitation beam resulting in depletion of emission from the outside ring of the excitation spot. A super-resolution image is generated by scanning the overlapping excitation beams over the sample.

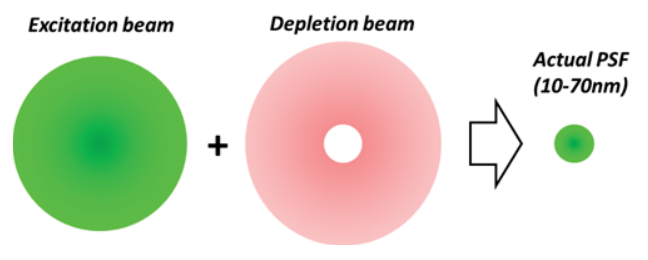

Near-field scanning optical microscopy (NSOM)
Excitation light is guided through a sub-wavelength aperture probe that is scanned over the sample. It relies on near-field excitation and requires close proximity of the NSOM probe (tapered optical fiber) and the sample.

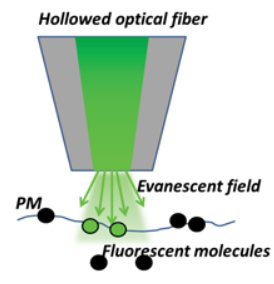

Limitations

Slow (data acquisition minutes to hours).

Requires additional equipment to achieve axial super resolution (e.g. interferometry PALM).

Highest resolution requires fixed samples.

Blinking and/or switching of organic dyes requires deoxygenated buffers. High lateral resolution (30-
$100 \mathrm{~nm})$.

FCS-STED is possible in living cells.

3D-imaging of sub-cellular structures.

Applicable to living specimens.
Involves expensive, specialized lasers and optics.

High intensity of the depletion beam.

Limited combination of fluorophores can be employed.

Dual-color imaging with equal spatial resolution is challenging.

\section{Scanning tip delivers a simultaneous topography map. \\ Multi-color imaging with equal resolution. \\ Limited evanescent field eliminates cytosolic background. \\ Perfectly suited to image apical cell membrane. \\ FCS-NSOM possible in living cells.}

Spatial resolution is dictated by probe diameter (70-90 nm).

Mostly used for fixed samples.

Technically challenging. Probe fragility.

dSTORM, direct STORM; FCS, fluorescence correlation spectroscopy; fPALM, fluorescence PALM; PALM, photoactivated localization microscopy; SPT, single-particle tracking; STED, stimulated emission depletion; STORM, stochastic optical reconstruction microscopy.

of lipid-linked protein nanoclusters. These accessory molecules include proteins associated with the actin cortex, the extracellular matrix and other molecules - including galectins - that have the capacity to bind galactose or galactose-derivative containing polysaccharides (Vasta, 2009). For example, intracellular galectins were shown to be important in stabilizing and enlarging GTP-loaded Ras nanoclusters (Belanis et al., 2008). In response to growth factor receptor activation, galectin-1 which has the capacity to bind intracellular prenyl groups translocates from the cytosol to the membrane and becomes important in recruiting GTP-loaded H-Ras to nanoclusters that do not require cholesterol and function as signal transduction platforms (Belanis et al., 2008; Prior et al., 2003). In this case, galectin-1 appears to replace cholesterol as the factor that stabilizes H-Ras-GTP nanoclusters. Moreover, when H-Ras is depalmitoylated, galectin-1 acts as a chaperone to transfer H-Ras to the Golgi.

In the case of GPI-APs, the cortical actin cytoskeleton must be considered because it can regulate the extent of nanoclustering (Goswami et al., 2008). This regulation can be either passive or active. In the first case, and as discussed in the Introduction, the membrane skeleton can be coupled to unspecified transmembrane proteins that act as 'picket fences' to create corrals in the plane of the membrane with dimensions that vary between 30 and $250 \mathrm{~nm}$ 
A

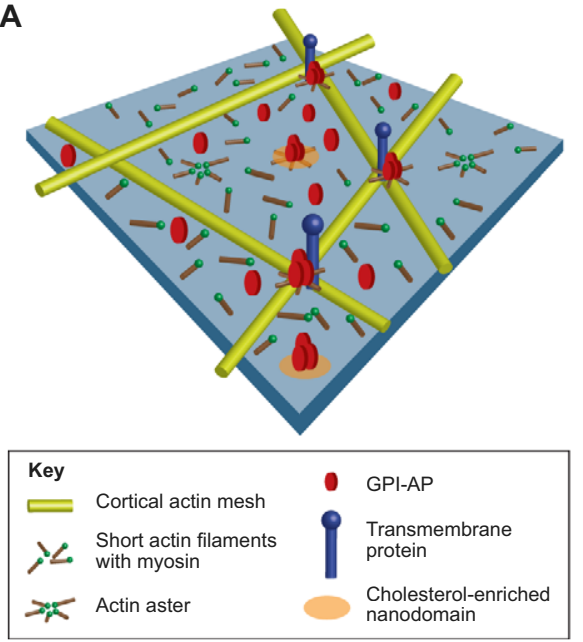

B

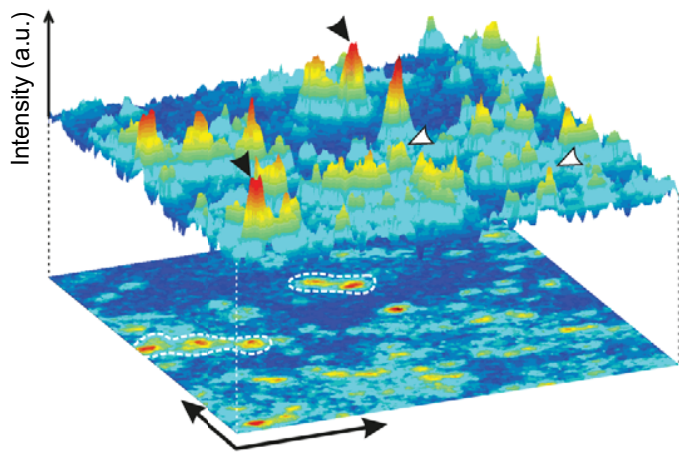

Fig. 1. Nanoscale organization of GPI-APs on the cell membrane. (A) The organization of GPI-APs on plasma membranes as co-existence between monomers and small nanoclusters containing a few molecules. Nanocluster formation appears to be driven by cortical actin (hypothetical actin asters) and

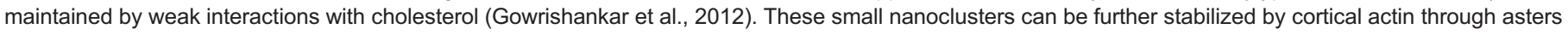
and/or proximal transmembrane proteins that act as 'posts' attached to the cortical actin. The physical separation between GPI-AP nanoclusters has been

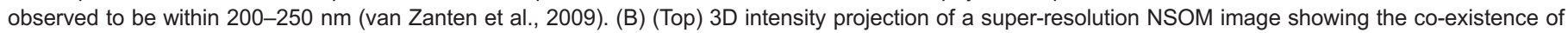
nanoclusters (black arrowheads) and monomers (white arrowheads) of GPI-APs. (Bottom) Areas encircled by a dashed line on the 2D image illustrate that

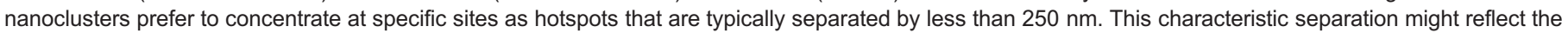

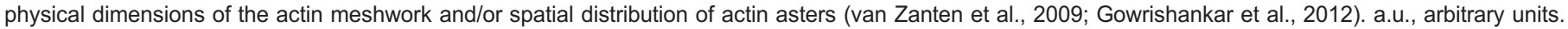
Image reproduced with permission (van Zanten et al., 2009).

depending on the cell type (Kusumi et al., 2011) (Fig. 1A). These fences could determine the upper limit on the size of nanoclusters by interposing a collection of transmembrane proteins acting as fences between adjacent nanoclusters.

It has been also proposed that dynamic, short actin filaments form aster-like aggregates under the plasma membrane that actively drive lipid-anchored proteins into nanoclusters (Gowrishankar et al., 2012) (Fig. 1A). The observation that GPI-AP nanoclusters are found to be spatially separated by a characteristic distance of less than $250 \mathrm{~nm}$ (van Zanten et al., 2009), further indicates that the assembly of actin asters close to the inner leaflet of the plasma membrane occurs at this spatial scale (Fig. 1B). However, a main unresolved issue is what gives rise to the coupling of GPI-AP nanoclusters in the outer leaflet with the sub-membranous actin cortex. One possible mechanism is that GPI-APs are coupled to complementary nanodomains (consisting of lipids, proteins or both) that reside in the inner membrane leaflet. These putative inner-leaflet nanodomains could associate with small, dynamic actin filaments in the cortex in a yet unknown fashion and actively draw GPI-APs into nanoclusters through myosin motors. Alternatively, GPI-AP nanoclusters could associate with transmembrane proteins that are coupled to the dynamic actin filaments.

Indeed, transmembrane proteins that are anchored to the cytoskeleton, either permanently or in a transient and regulated manner, might also serve as 'posts' that stabilize GPIAP nanoclusters through cis interactions they undergo with nanocluster components. This mechanism was, in fact, originally proposed to explain how membrane proteins are linked to the underlying membrane-associated cytoskeleton, as observed during patching and capping (Singer, 1977). This is a phenomenon in which membrane proteins that have been crosslinked by antibodies passively form patches that are subsequently actively cleared to a distal part of the cell surface through coupling to a retrograde cortical actin flow. However, a key issue is the identity of the transmembrane proteins involved and how the linkage to the cytoskeleton might be regulated. In this regard, GPI-AP nanoclusters that have been induced by antibody-coated, multivalent gold particles undergo transient anchorage (Chen et al., 2006), whereby their lateral diffusion is arrested for several seconds before they become mobile again. Moreover, this transient anchorage is dependent on cholesterol and one of the seven transmembrane adaptor proteins (TRAPs), namely CBP (officially known as PAG1) (Hořejší et al., 2004). $\mathrm{CBP}$ is involved in anchoring GPI-AP nanoclusters in a manner that depends on phosphorylation and the cytoskeletal adaptor ERM-binding protein 50 (EBP50; officially known as SLC9A3R1), which binds to members of the ezrin-radixinmoesin (ERM) family (Chen et al., 2009). Presumably, the CBPEBP50-ERM complex could link a GPI-AP nanocluster to the underlying actin cytoskeleton. A similar transient anchorage, probably also mediated by components of the membraneassociated cytoskeleton, termed stimulated temporary arrest of lateral diffusion (STALL), has been observed for ligand-induced nanoclusters of the GPI-AP CD59. Moreover, these STALL locations were associated with downstream intracellular signaling processes (Suzuki et al., 2007).

Recent two-color super-resolution studies also suggest that the cortex has an important role because enhanced cortical actin was found to be associated with nanodomains of the influenza envelope transmembrane protein hemagglutinin (HA) (Gudheti et al., 2013). This association could limit the lateral mobility of $\mathrm{HA}$ in actin-enriched regions. Moreover, the authors were able to alter domain size and HA density within the domain by using reagents that perturb F-actin. In addition, the actin-binding and actin-disassembling factor cofilin is predominantly excluded from actin-enriched regions that underlie the HA nanodomains, suggesting that HA and/or proteins within the HA cluster mediate this exclusion (Gudheti et al., 2013). All of the studies described above not only demonstrate that the actin cortex is an 
important determinant of nanocluster organization but also that nanoclusters have the potential to regulate the distribution of actin-binding proteins in the underlying cortex.

\section{Transmembrane proteins involved in the immune system}

In addition to lipid-linked proteins, many transmembrane proteins also form nanoclusters. Below, we will discuss examples of transmembrane protein nanoclusters with relevance for immunological processes.

\section{LFA-1}

The main integrin receptor involved in leukocyte adhesion is the lymphocyte function-associated antigen-1 (LFA-1, officially known as ITGAL), which forms nanoclusters of $\sim 70 \mathrm{~nm}$ on monocytes prior to ligand binding (Cambi et al., 2006). The integrity of these nanoclusters is independent of cholesterol or the actin cytoskeleton and the clusters contain on average six LFA-1 molecules (Cambi et al., 2006). By using NSOM, a first level of hierarchy for LFA-1 organization was defined because ligandindependent LFA-1 nanoclusters resided within $50-100 \mathrm{~nm}$ of GPI-AP nanodomains (van Zanten et al., 2009) (Fig. 2). Upon ligand-induced LFA-1 activation, a higher level of hierarchy that is associated with function was observed (van Zanten et al., 2009). Indeed, coalescence of the two nanodomain types and further recruitment of GPI-APs to these sites resulted in the formation of stable cell adhesion nanoplatforms (van Zanten et al., 2009) (Fig. 2). Although there is no clear explanation yet with regard to what keeps LFA-1 in nanoclusters, it is tempting to speculate that LFA-1 and GPI-AP nanoclusters are somehow interconnected by the actin cytoskeleton to the sub-membranous actin asters described above (Gowrishankar et al., 2012). More recently, using a combination of NSOM and SPT, the importance of the actin cytoskeleton was demonstrated for inside-out activation of LFA-1, its stable ligand binding and firm cell adhesion (Bakker et al., 2012). Although LFA-1 nanoclustering in resting monocytes was observed prior to ligand activation, it is worth mentioning that other integrins, such as $\beta_{1}$ and $\beta_{3}$, form nanoclusters upon ligand activation (Rossier et al., 2012; Yu et al., 2012). These nascent nanoclusters are thought to play a key role in the initiation of cell adhesion (Yu et al., 2012) and in the formation of focal adhesion complexes (Rossier et al., 2012; van Zanten et al., 2009; Yu et al., 2012).

\section{DC-SIGN}

The pathogen recognition receptor dendritic cell-specific ICAM-3-grabbing non-integrin 1 (DC-SIGN; officially known as CD209) binds an astonishingly large variety of human infectious agents ranging from HIV to dengue viruses to yeasts. It is found on the surface of immature dendritic cells for the purpose of subsequent antigen presentation. Biochemical studies have shown that DC-SIGN predominantly forms tetramers (Feinberg et al., 2005; Serrano-Gómez et al., 2008; Yu et al., 2009). Recently, work from our laboratories using EM and different super-resolution approaches demonstrated that DCSIGN forms nanoclusters on immature dendritic cells (or following ectopic expression in rodent cells) prior to pathogen
A

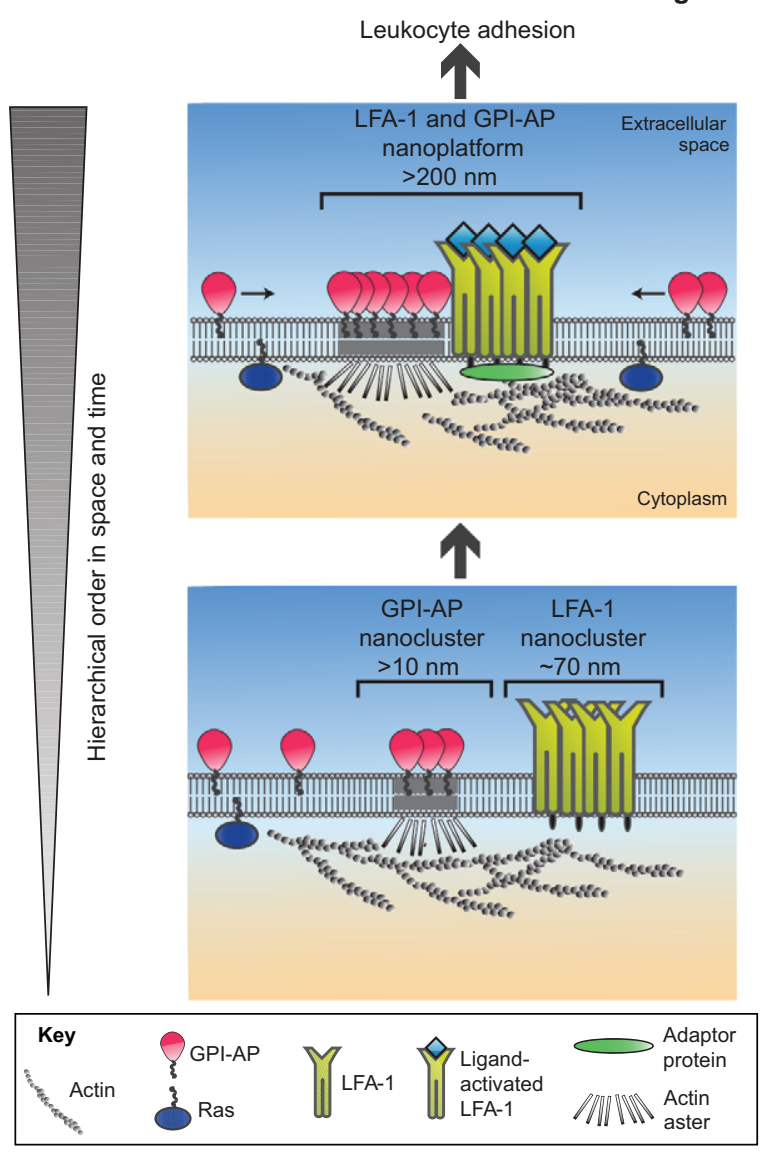

B

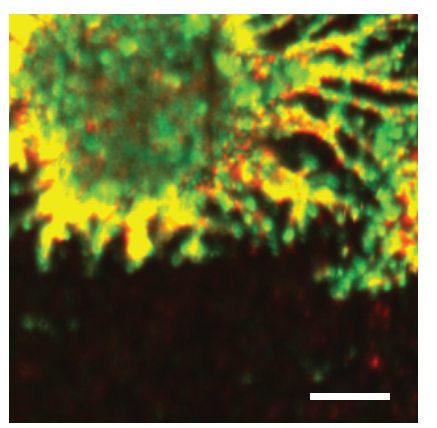

$\uparrow$

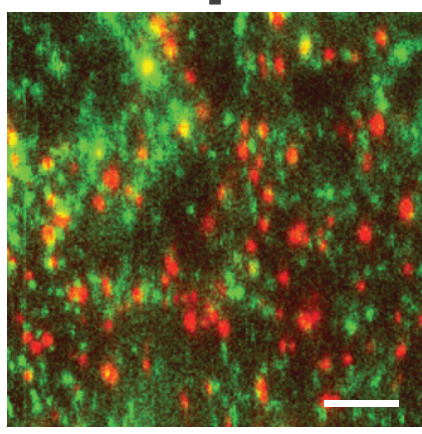

Fig. 2. Hierarchical organization of GPI-APs and the cell adhesion integrin receptor LFA1. The inverted triangle at the left indicates the increase in hierarchical order in space and time from bottom to top of each panel. (A) Bottom panel: GPI-AP nanoclusters (containing two to four molecules in total) and pre-formed LFA-1 nanoclusters (six to ten molecules in total) have been observed in close proximity from each other $(50-150 \mathrm{~nm}$ ) before LFA-1 is activated through ligand binding. Top panel: Activation of LFA-1 through ligand binding, correlates with an increase in the number of GPI-AP molecules in each nanocluster that is likely to be mediated by local rearrangements of the cytoskeleton through adaptor proteins (green). Furthermore, incorporation (arrows) of mobile monomeric GPI-APs and diffusible LFA-1 nanoclusters (Bakker et al., 2012) can further strengthen the nanoclusters and lead to the assembly of LFA-1 and GPI-AP-containing nanoplatforms that are adhesion competent. (B) Bottom panel:

Representative super-resolution NSOM images of GPI-AP (green) and LFA-1 nanoclusters (red) in resting monocytes show that LFA-1 and GPIAP are not associated but in close proximity to each other. Scale bar: $1 \mathrm{~mm}$. Top panel: Ligand activation of LFA-1 leads to aggregation of GPIAPs and LFA-1 (visualized by the substantial increase in yellow areas, which indicates spatial colocalization at the nanoscale) into adhesioncompetent nanoplatforms. Scale bar: $5 \mu \mathrm{m}$. Images have been taken with permission from van Zanten et al., 2009. 
A

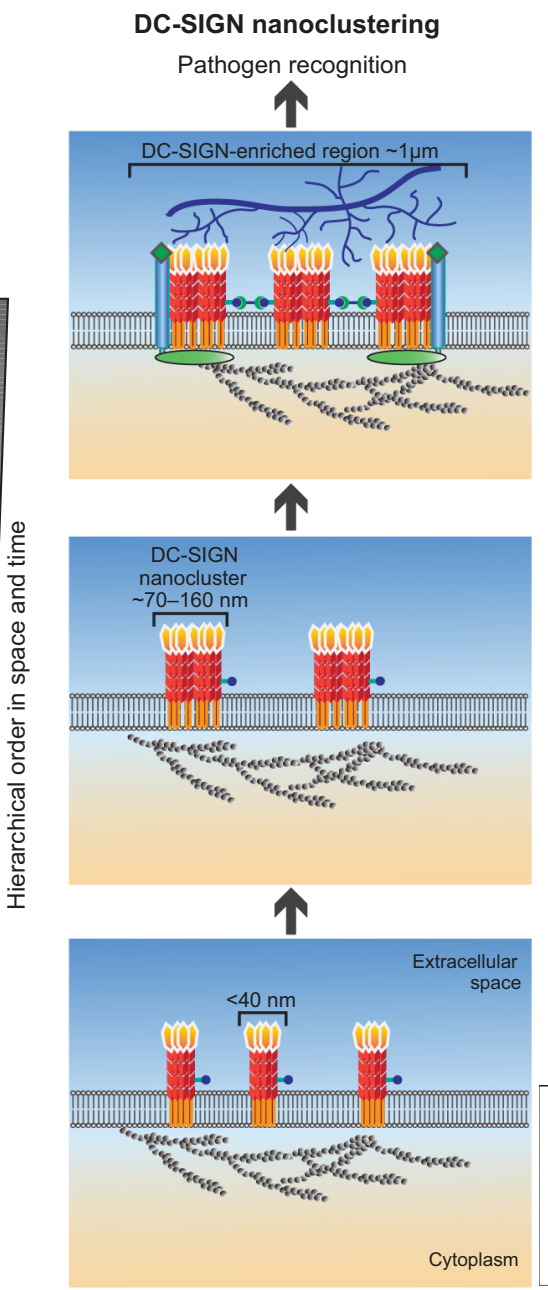

B
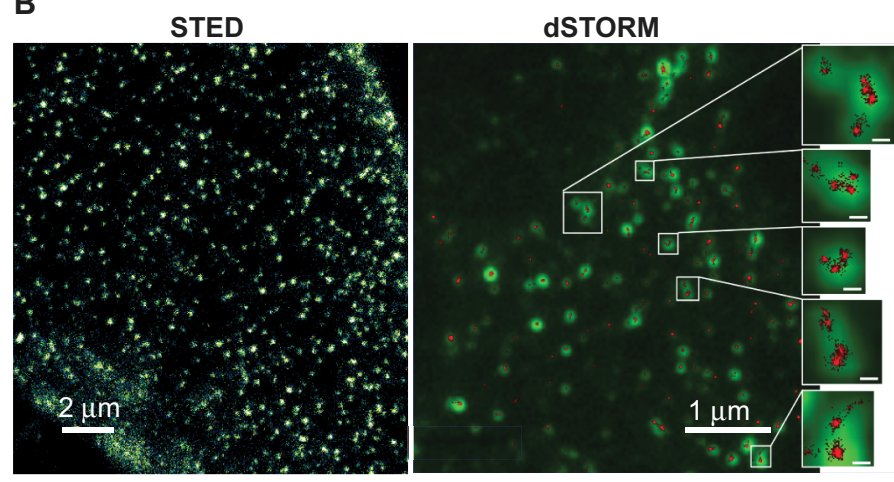

DC-SIGN and clathrin
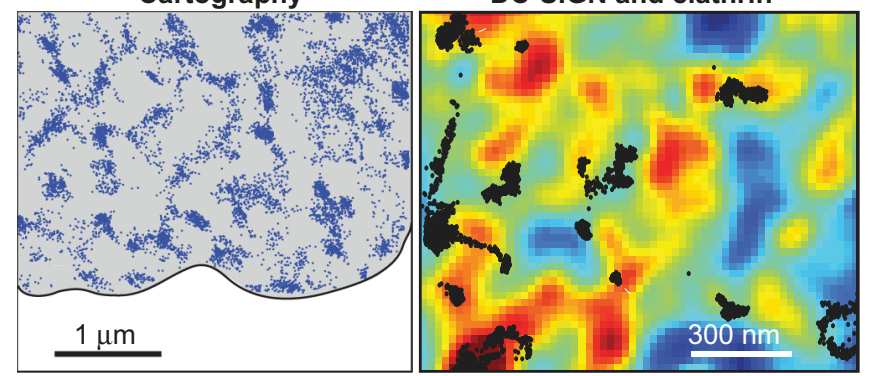

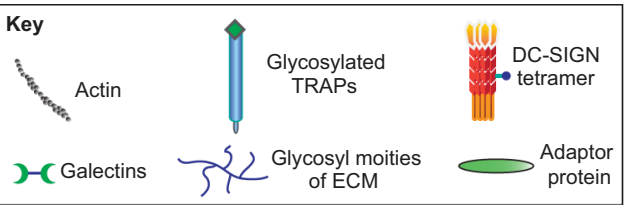

Fig. 3. Hierarchical organization of the pathogen recognition receptor DC-SIGN. The inverted triangle to the left indicates the increase in hierarchical order in space and time from bottom to top of each panel. (A) DC-SIGN is thought to be expressed predominantly in the form of tetramers (bottom panel) on the cell surface. These then aggregate further and form DC-SIGN nanoclusters (middle panel). Nanoclusters, in turn, are recruited to specialized regions of the cell membrane (top panel) and maintained through a number of additional interactions with, e.g. TRAPs (see text). (B) Representative images from different superresolution and SPT methods illustrate the highly hierarchical organization of DC-SIGN. The stimulated emission-depletion (STED) microscopy image (top left), with a resolution of $\sim 90 \mathrm{~nm}$, clearly shows DC-SIGN nanoclusters. As shown in the enlarged regions of the direct stochastic optical reconstruction microscopy (dSTORM) image (top right), nanoclusters appear to be in close proximity to each other. The cartography map (bottom left) represents the reconstructed molecular positions (blue dots) obtained from single-particle tracking (SPT) movies of several DC-SIGN nanoclusters as they explore the cell membrane (Torreno-Pina et al., 2014). This map demonstrates that DC-SIGN nanoclusters explore restricted areas of $\sim 1 \mu \mathrm{m}$, which is consistent with static dSTORM images. Superimposition of a DC-SIGN map with images of clathrin illustrate that DC-SIGN compartments are enriched with clathrin (bottom right). Black areas indicate the position of DC-SIGN, the colored background represents the intensity of the clathrin signal from low (blue) to high (red) intensity. Adapted with permission from Torreno-Pina et al., 2014.

binding (Fig. 3). The nanoclusters vary in size from 70-160 nm and contain around ten molecules per nanocluster (Cambi et al., 2004; de Bakker et al., 2007; Itano et al., 2012; Liu et al., 2014; Manzo et al., 2012).

Interestingly, the 'neck' region of DC-SIGN is presumably not only responsible for tetramer formation (Serrano-Gómez et al., 2008) but also, in part, for its nanoclustering, indicating that its organization on the cell membrane is also hierarchical (Manzo et al., 2012). Furthermore, mutation studies suggested that ectodomain interactions that involve the carbohydrate recognition domain (CRD) of DC-SIGN serve to stabilize nanoclusters (Liu et al., 2012). DC-SIGN organization in nanoclusters has been shown to be crucial for enhancing its binding capability to small pathogens such as viruses (Cambi et al., 2004; Manzo et al., 2012), indicating that hierarchy in organization correlates with function. There are several possibilities for how DC-SIGN nanoclusters can become stabilized (Liu et al., 2012). The DC-SIGN CRD could bind to glycosaminoglycans or glycosyl moieties of ECM proteins, or to transmembrane proteoglycans that link to the ECM or to TRAPs, which might be directly or indirectly linked to the membraneapposed cytoskeleton. More recently, we found that DC-SIGN nanoclusters further organize into larger compartments of $\sim 1 \mu \mathrm{m}$ in size that are maintained by interactions between the receptor glycosylation motif and surface galectins (Torreno-Pina et al., 2014) (Fig. 3). These regions appeared enriched in the endocytic vesicle coat-component clathrin, and its interactions with DCSIGN are highly affected by the glycan network. As such, cell surface glycan-mediated interactions might represent another, previously unidentified, layer of cell membrane organization at 
the microscale that could have functional relevance in regulating clathrin-mediated endocytosis of the receptor and its bound ligands. Given that many membrane receptors are glycosylated, it is conceivable that glycan interactions have a role in the spatiotemporal organization of other receptors, in a manner that is similar to what has been observed for DC-SIGN.

T-cell receptors, linkers for activation of T cells and B-cell receptors Several other receptors involved in the immune system have also been shown to form nanoclusters (Schamel and Alarcón, 2013). For instance, there is accumulating evidence that T-cell receptors (TCRs) form nanoclusters on the surface of unstimulated T cells. Initial biochemical and EM experiments carried out in Alarcon's group showed that monomers and TCR nanoclusters co-exist on resting $\mathrm{T}$ cells (Schamel et al., 2005). Another study confirmed this by using the two-color coincidence-detection technique but found a smaller population of nanoclusters compared to monomers (James et al., 2007). More recently, TCR nanoclustering has been demonstrated by EM and PALM on fixed cells (Lillemeier et al., 2010). Although the molecular mechanisms for the formation of TCR nanoclusters are still highly debated, nanocluster stability appears to depend on cholesterol and sphingomyelin (Molnár et al., 2012; Schamel et al., 2005). Because $\mathrm{T}$ cells can regulate the extent of TCR nanoclustering depending on their activity, i.e. naïve versus memory $\mathrm{T}$ cells, it has been suggested that nanoclustering facilitates a quick memory response upon T-cell activation (Kumar et al., 2011).

The adaptor proteins linker for activation of T-cells 1 and 2 (LAT1 and LAT2, hereafter referred to as LAT) are members of the TRAP family (Hořejší et al., 2004) and also form nanoclusters on resting $\mathrm{T}$ cells, although the size of these nanoclusters and their participation in T-cell signaling are still debated. Lillemeier et al. showed that TCR and LAT exist as separate and discrete nanoclusters (also termed 'protein islands' by the authors) 70$140 \mathrm{~nm}$ in diameter that concatenate (without mixing) during

TCR and LAT nanoclustering

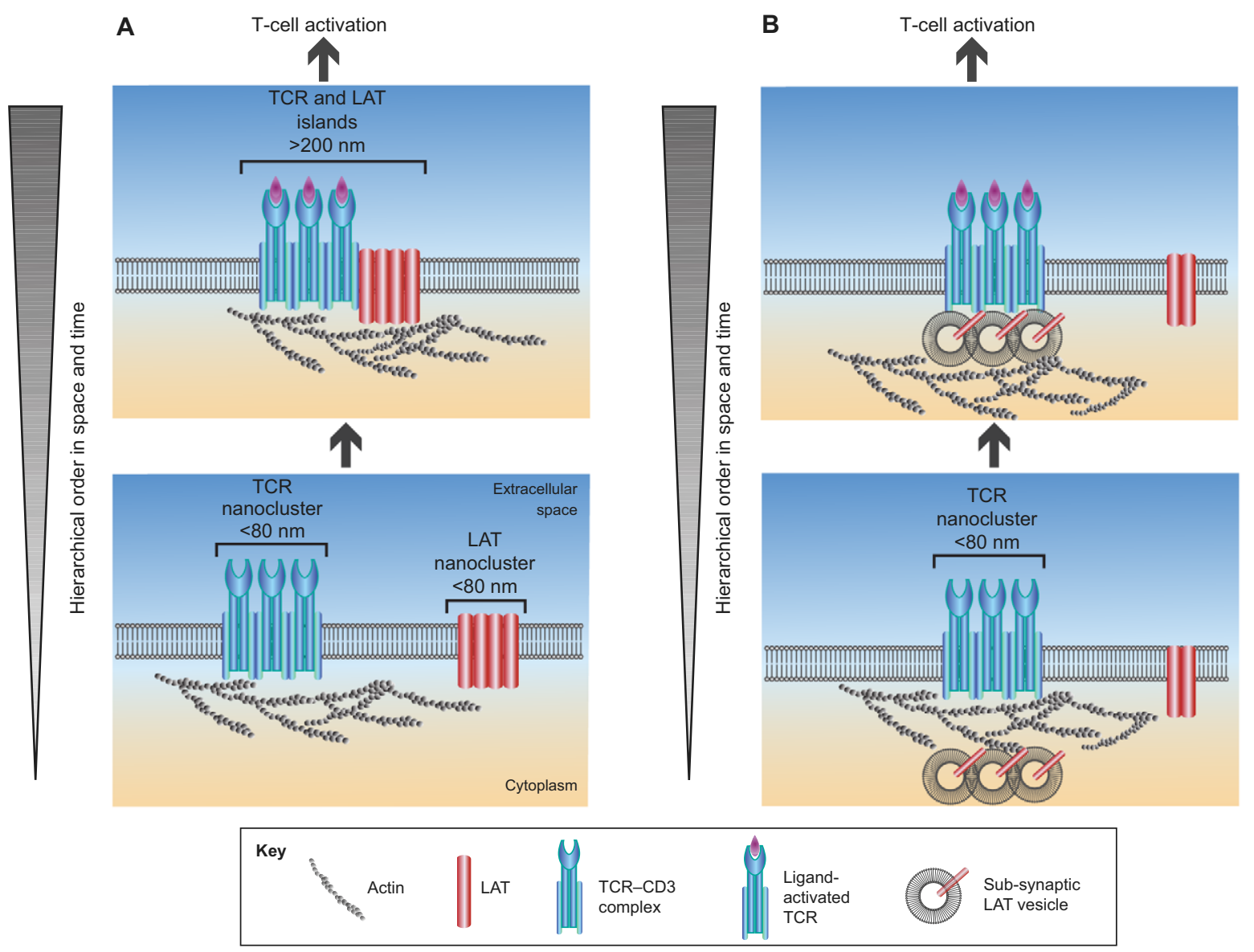

Fig. 4. Two possible pathways for the hierarchical organization of TCR and LAT molecules at T cells. The inverted triangle to the left indicates the increase in hierarchical order in space and time from bottom to top of each panel. (A) One possible pathway considered in the field, termed the 'protein island' model. TCR-CD3 [comprising TCR and the T-cell co-receptor cluster of differentiation 3 (CD3), also known as Cde3] complexes and LAT molecules are already present as small preassembled nanoclusters on the surface of resting T cells (bottom panel). After antigen recognition and TCR activation, TCR-CD3 and LAT nanoclusters concatenate, but do not mix (top panel). The actin cytoskeleton is thought to play a main role in this process (Lillemeier et al., 2010). Evidence for the protein island model comes from EM and PALM images before and after TCR activation. (B) An alternative assembly pathway of TCR and LAT nanoclusters, involving sub-synaptic LAT vesicles. TCR-CD3 complexes, as well as some LAT molecules, exist as small nanoclusters on the surface of resting T cells (bottom panel). LAT can also be found in sub-synaptic vesicles (top panel). After TCR activation, only LAT molecules within the sub-synaptic vesicles in close proximity to the cell membrane participate in signal transduction (Williamson et al., 2011). Evidence for the involvement of LAT sub-synaptic vesicles comes from PALM images in living cells, which show LAT recruitment in close proximity to the cell membrane. These LAT nanoclusters appear and disappear quickly over time, suggesting that vesicles dock and undock at the membrane without undergoing any appreciable lateral movement (Williamson et al., 2011). 
activation (Lillemeier et al., 2010) (Fig. 4A). By contrast - using similar super-resolution approaches - another study showed that TCR and LAT nanoclusters are highly mixed, and that LAT nanoclusters are composed of only a few molecules (Sherman et al., 2011). These nanoclusters might not participate in early Tcell signaling events because sub-synaptic vesicles of LAT were seen to dock to the plasma membrane to TCR-activation sites (Williamson et al., 2011) (Fig. 4B), a process that is dependent on the vesicle (v)-SNARE protein VAMP7 (Larghi et al., 2013). The reasons for these contrasting results are not yet clear but they might be related to differences in the TCR activation times used by the different groups.

The multi-chain high-affinity $\operatorname{IgE} \varepsilon$ receptor subunit $\beta$ (FceRI; also known as MS4A2) and BCR are other examples of receptors that pre-cluster prior to antigen binding (Veatch et al., 2012; Wilson et al., 2001; Yang and Reth, 2010). For instance, by using a combination of direct stochastic optical reconstruction microscopy (dSTORM) and SPT, BCR and its co-receptor CD19 were found to exist in spatially distinct preformed nanoclusters in resting B cells (Mattila et al., 2013). Although the actin cytoskeleton regulates BCR, CD19 mobility appears restricted by the tetraspanin CD81, indicating that both CD19 and CD81 can form scaffolds to support BCR signaling (Mattila et al., 2013).

\section{Outstanding investigations - biogenesis, formation and stability of nanoclusters}

The above examples illustrate that nanoclustering is a common organizing principle for many membrane receptors, although the clusters might be subject to different regulatory mechanisms, depending on the particular receptor involved. Nevertheless, the prevalence of nanoclustering has raised a number of important questions that have not yet been fully answered, such as any that relate to biogenisis, formation and stability of nanoclusters as well as the potential role of the non-clustered molecules.

\section{Nanocluster biogenesis, formation and stability}

The full intracellular molecular complement of membrane nanoclusters (e.g. individual protein monomers, preformed protein homo-oligomers, preformed protein hetero-oligomers and/or particular lipid components) is typically unknown. Of interest here is whether a given nanocluster arrives at the plasma membrane as an almost fully assembled entity - as appears to be the case for LAT (Williamson et al., 2011) - with only a few factors to be subsequently recruited and/or eliminated or, by contrast, almost completely unassembled. It is unlikely that most membrane nanoclusters are composed solely of close-packed, identical proteins, although the idea is conceptually attractive. Instead, the current evidence strongly suggests that a specific complement of lipids and (most probably) other proteins will be present in nanoclusters. Indeed, at least in the case of DC-SIGN (Itano et al., 2011; Itano et al., 2012), LFA-1 (van Zanten et al., 2009) and BCR (Mattila et al., 2013), nanoclusters are not fully packed with their respective receptors.

The mechanism by which nanocluster precursors undergo initial reorganization after their delivery to the plasma membrane is a key issue. One might expect at least passive reorganization to occur in response to the differences in chemical composition between the intracellular and plasma membrane environments. These arise from at least three specific characteristics of plasma membranes: (1) molecular components have specific orientations and cannot be treated as soluble proteins with the freedom to reorient in three dimensions; (2) the environment contains additional structural features, i.e. the outer and inner membrane environments are considerably different; and (3) the membrane is very crowded. Involvement of an active rather than passive process in the formation of nanoclusters and their stabilization is also possible (Gowrishankar et al., 2012).

If the mechanisms of nanocluster formation involve at least the partial self-association at the plasma membrane, then how are these associations limited to the nanoscale? The limiting factors could be of biochemical nature (i.e. they might constitute specific molecular interactions), reflect cell biological aspects (e.g. transmembrane pickets attached to the cytoskeleton) (Kusumi et al., 2011) or result from physical effects (e.g. kinetics trapping or the entropic costs associated with nanocluster existence). Importantly, at least in several cases, there is no particular size limit; instead nanoclusters of varying sizes simultaneously coexist (de Bakker et al., 2007; Gudheti et al., 2013; Itano et al., 2012; Williamson et al., 2011).

Elucidating the factors that contribute to nanocluster stability is crucially important in understanding nanocluster function and regulation. Generally speaking, nanoclusters could merge, partially disassemble, change their biochemical composition or completely dissolve. A dynamic equilibrium may also exist between nanoclusters and their components in the nearby membrane environment; for instance, although DC-SIGN molecules in nanoclusters do not appreciably exchange with those in the surrounding membrane, HA molecules in nanoclusters do (Itano et al., 2011). Another characteristic that is intrinsically related to nanoclusters is their lateral mobility within the membrane plane. Examples, of functions that require nanocluster mobility, are the transport of complexes between viruses and DC-SIGN nanoclusters to sites of cellular internalization (van der Schaar et al., 2007), rearrangement of the relative spatial distributions of TCR and LAT nanoclusters after T cell activation (Lillemeier et al., 2010), and rearrangement and further recruitment of GPI-APs to activated LFA-1 nanoclusters (van Zanten et al., 2009). Finally, the role of the membrane-associated actomyosin cell cortex has only just began to be investigated in detail, both structurally and functionally (Gowrishankar et al., 2012; Gudheti et al., 2013; Kusumi et al., 2011; Luo et al., 2013; Xu et al., 2013). Determination of its actual organization and structural association with membrane components should be an area of intense investigation in the future.

\section{Role of non-clustered components}

Although the forces that drive and limit nanocluster assembly are still under discussion, it is important to note that clustering is rarely complete and that, in many cases, nanoclusters co-exist with randomly distributed non-clustered components, as shown for TCR, LAT, GPI-APs, CD71 (van Zanten et al., 2009) and CD36 (Jaqaman et al. 2011), suggesting that such co-existence has a functional or regulatory role (Fig. 5). For example, nanoclusters could act as pre-assembled units that have the potential to become activated upon ligand binding because they spatially concentrate receptors and commonly exhibit restricted diffusion or are even spatially trapped. Yet, they might exist below a functional threshold because of their small size and, as such, are not able to stably bind their ligands. Alternatively, binding of ligands to such small nanoclusters might not be sufficient to elicit a downstream response. Non-clustered components, typically characterized by faster and unconstrained diffusion, might modulate nanocluster function by being 


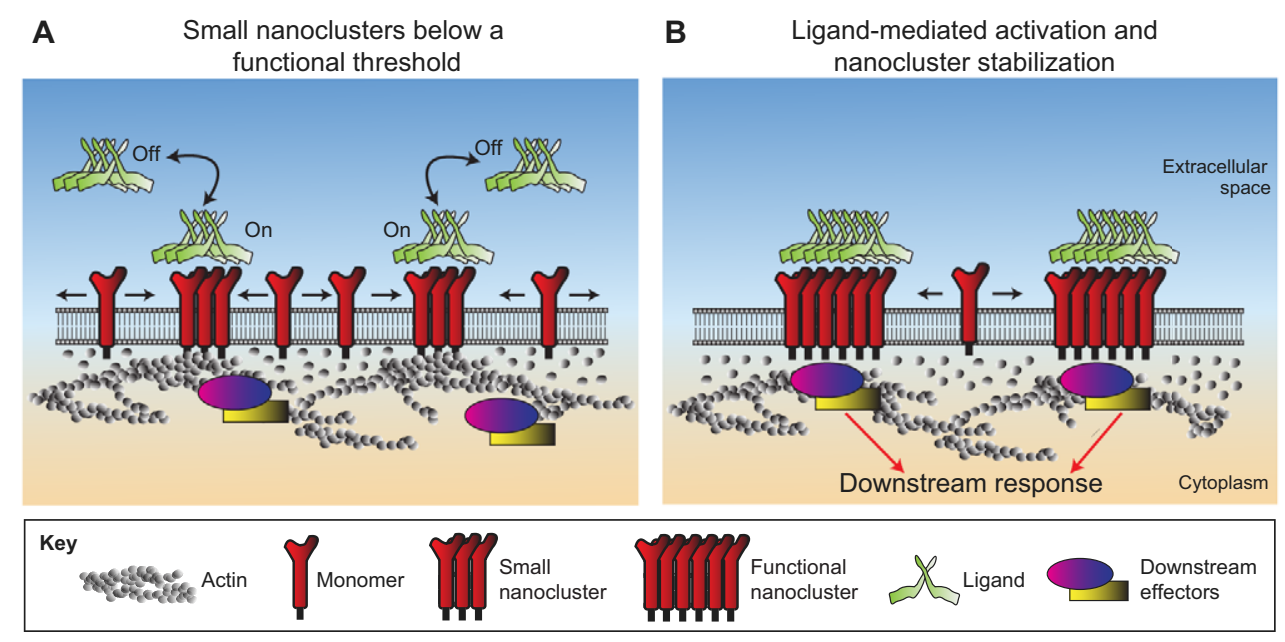

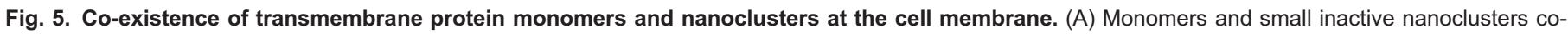

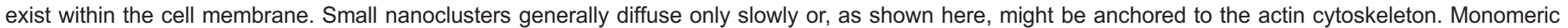

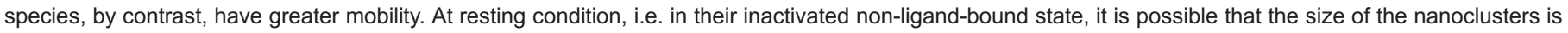
below a functional threshold, at which they cannot stably bind their ligands (the on-off arrows indicate an equilibrium between extracellular and bound

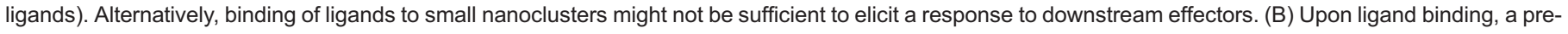
existing small nanocluster can incorporate further monomers. Nanocluster activation through ligand binding and further recruitment of protein monomers

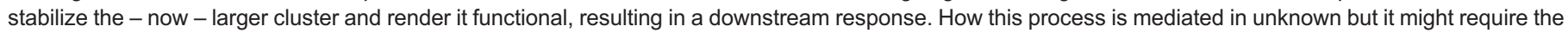

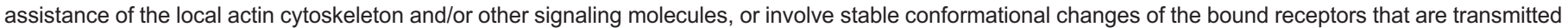
to the monomer species.

incorporated into pre-existing nanoclusters, thereby acting as switches that provide the necessary threshold of nanocluster assembly in order to initiate their function. According to this hypothesis, dynamic pre-formed nanoclusters may not be functional when their size is below a specific threshold. Nonclustered receptors could provide a positive feedback to create larger domains as a way to trigger nanocluster assembly and hierarchical organization in a functional context. Such a positive feedback would, in principle, come into play upon nanocluster activation, and be mediated by lateral diffusion and encounter with nanoclusters, as recently suggested for TCRs (Schamel and Alarcón, 2013).

\section{Why nanoclusters?}

The ubiquitous presence of protein nanoclusters in mammalian plasma membranes prompts the crucial question of why nature has chosen this feature. Possible answers might come from elucidating why many soluble proteins are found in oligomeric states. This fundamental question has been addressed in several reviews that discuss plausible hypotheses, many of which may also be applicable to membrane protein nanoclusters (Ali and Imperiali, 2005; Hashimoto et al., 2011; Lynch, 2013). One important feature of soluble, oligomeric proteins is that they present the possibility of allosteric regulation; thus membrane protein nanoclusters might also be subject to this type of regulation. Another potential function of oligomerization of soluble proteins is to provide multiple, identical, ligand-binding sites in close proximity. For multivalent ligands, nanoclustering might increase the effective ligand-binding affinity (Cebecauer et al., 2010). For example, DC-SIGN nanoclusters recognize and bind to a large variety of glycosylated pathogens, and this ability might not only depend on the monosaccharide-binding ability of its CRD but also on the multivalency of the receptor within nanoclusters. Furthermore - compared with non-clustered receptors - in the case of monovalent ligands, oligomerization of their receptors can result in a higher dwell time of the ligand because ligand rebinding is facilitated (Lagerholm and Thompson, 1998). Moreover, the time of ligand engagement to the nanocluster might be regulated by altering the density of clustered ligand-binding sites because ligand-rebinding probability increases with the square of the binding-site density.

With respect to signal transduction, Hancock and co-workers (Harding and Hancock, 2008; Tian et al., 2007) have proposed that nanoclustering in the plasma membrane offers a general way to 'digitalize' analog input signals according to their strength by increasing the number of nanoclusters, because each of these provides a discrete switch-like output because of the short lifetime of nanoclusters (Murakoshi et al., 2004). For example, in response to EGF stimulation, ligation of the EGF receptor activates K-Ras, thereby generating nanoclusters that provide digital inputs to the Ras-Raf-MEK-ERK kinase signaling cascade. This allows the pathway to be sensitive to small amounts of the activating ligand. The 'quantal' outputs from each signaling nanocluster are 'counted', i.e. integrated, in the cytoplasm by the kinase cascade to generate the bulk, analog cellular response.

\section{Conclusions and perspectives}

A number of recent studies have demonstrated that membrane protein nanoclusters and their functional remodeling is an emerging theme in eukaryotic plasma membrane organization. It is reasonable to expect that, as the field progresses particularly in light of the increasing availability of superresolution microscopy - even more examples will be discovered. The development of technologies that permit simultaneous, multiplexed observation of different nanocluster types in living cells might, in the near future, give rise to a new field of systems nanobiology to specifically address the structural and functional properties of membrane protein nanoclusters. Although each imaging technique has potential drawbacks, the convergence of 
observations obtained with different approaches strongly suggests that nanoclustering will be increasingly recognized as a predominant feature. One of the challenges that lies ahead is to determine with certainty why nanoclustering is so prevalent.

\section{Acknowledgements}

We apologize to all those investigators whose work we could not cite due to space limitations. STED microscopy images of DC-SIGN were obtained at the ICFO's Super-Resolution Light Nanoscopy Facility, SLN@ICFO.

\section{Competing interests}

The authors declare no competing interests.

\section{Funding}

A.C. is the recipient of a NWO Meervoud subsidy. This work was supported by the National Institute of Health (K.J. and N.T), the European Commission (M.F.G.-P. and A.C.), Laserlab-Europe (M.F.G.-P.), the Human Frontiers Science Program (M.F.G.-P. and A.C.); and the Spanish Ministry of Science (M.F.G.-P. and J.A.T.-P). Deposited in PMC for release after 12 months.

\section{References}

Ali, M. H. and Imperiali, B. (2005). Protein oligomerization: how and why. Bioorg Med. Chem. 13, 5013-5020.

Anderson, R. G. W. and Jacobson, K. (2002). A role for lipid shells in targeting proteins to caveolae, rafts, and other lipid domains. Science 296, 1821-1825.

Bakker, G. J., Eich, C., Torreno-Pina, J. A., Diez-Ahedo, R., Perez-Samper, G., van Zanten, T. S., Figdor, C. G., Cambi, A. and Garcia-Parajo, M. F. (2012) Lateral mobility of individual integrin nanoclusters orchestrates the onset for leukocyte adhesion. Proc. Natl. Acad. Sci. USA 109, 4869-4874.

Belanis, L., Plowman, S. J., Rotblat, B., Hancock, J. F. and Kloog, Y. (2008). Galectin-1 is a novel structural component and a major regulator of h-ras nanoclusters. Mol. Biol. Cell 19, 1404-1414.

Cambi, A., de Lange, F., van Maarseveen, N. M., Nijhuis, M., Joosten, B., van Dijk, E. M. H. P., de Bakker, B. I., Fransen, J. A. M., Bovee-Geurts, P. H. M., van Leeuwen, F. N. et al. (2004). Microdomains of the C-type lectin DC-SIGN are portals for virus entry into dendritic cells. J. Cell Biol. 164, 145155.

Cambi, A., Joosten, B., Koopman, M., de Lange, F., Beeren, I., Torensma, R., Fransen, J. A., Garcia-Parajó, M., van Leeuwen, F. N. and Figdor, C. G. (2006). Organization of the integrin LFA-1 in nanoclusters regulates its activity. Mol. Biol. Cell 17, 4270-4281.

Cebecauer, M., Spitaler, M., Sergé, A. and Magee, A. I. (2010). Signalling complexes and clusters: functional advantages and methodological hurdles. J. Cell Sci. 123, 309-320.

Chen, Y., Thelin, W. R., Yang, B., Milgram, S. L. and Jacobson, K. (2006) Transient anchorage of cross-linked glycosyl-phosphatidylinositol-anchored proteins depends on cholesterol, Src family kinases, caveolin, and phosphoinositides. J. Cell Biol. 175, 169-178.

Chen, Y., Veracini, L., Benistant, C. and Jacobson, K. (2009). The transmembrane protein $\mathrm{CBP}$ plays a role in transiently anchoring small clusters of Thy-1, a GPI-anchored protein, to the cytoskeleton. J. Cell Sci. 122, 3966-3972.

de Bakker, B. I., de Lange, F., Cambi, A., Korterik, J. P., van Dijk, E. M. H. P., van Hulst, N. F., Figdor, C. G. and Garcia-Parajo, M. F. (2007). Nanoscale organization of the pathogen receptor DC-SIGN mapped by single-molecule high-resolution fluorescence microscopy. Chemphyschem 8, 1473-1480.

Delaguillaumie, A., Harriague, J., Kohanna, S., Bismuth, G., Rubinstein, E., Seigneuret, M. and Conjeaud, H. (2004). Tetraspanin CD82 controls the association of cholesterol-dependent microdomains with the actin cytoskeleton in T lymphocytes: relevance to co-stimulation. J. Cell Sci. 117, 5269-5282.

Eggeling, C., Ringemann, C., Medda, R., Schwarzmann, G., Sandhoff, K., Polyakova, S., Belov, V. N., Hein, B., von Middendorff, C., Schönle, A. et al. (2009). Direct observation of the nanoscale dynamics of membrane lipids in a living cell. Nature 457, 1159-1162.

Feinberg, H., Guo, Y., Mitchell, D. A., Drickamer, K. and Weis, W. I. (2005). Extended neck regions stabilize tetramers of the receptors DC-SIGN and DC SIGNR. J. Biol. Chem. 280, 1327-1335.

Friedrichson, T. and Kurzchalia, T. V. (1998). Microdomains of GPI-anchored proteins in living cells revealed by crosslinking. Nature 394, 802-805.

Goswami, D., Gowrishankar, K., Bilgrami, S., Ghosh, S., Raghupathy, R., Chadda, R., Vishwakarma, R., Rao, M. and Mayor, S. (2008). Nanoclusters of GPI-anchored proteins are formed by cortical actin-driven activity. Cell 135, 1085-1097.

Gowrishankar, K., Ghosh, S., Saha, S., C, R., Mayor, S. and Rao, M. (2012). Active remodeling of cortical actin regulates spatiotemporal organization of cell surface molecules. Cell 149, 1353-1367.

Gudheti, M. V., Curthoys, N. M., Gould, T. J., Kim, D., Gunewardene, M. S., Gabor, K. A., Gosse, J. A., Kim, C. H., Zimmerberg, J. and Hess, S. T. (2013). Actin mediates the nanoscale membrane organization of the clustered membrane protein influenza hemagglutinin. Biophys. J. 104, 21822192.

Harding, A. S. and Hancock, J. F. (2008). Using plasma membrane nanoclusters to build better signaling circuits. Trends Cell Biol. 18, 364-371.

Hashimoto, K., Nishi, H., Bryant, S. and Panchenko, A. R. (2011). Caught in self-interaction: evolutionary and functional mechanisms of protein homooligomerization. Phys. Biol. 8, 035007.

Hořejší, V., Zhang, W. and Schraven, B. (2004). Transmembrane adaptor proteins: organizers of immunoreceptor signalling. Nat. Rev. Immunol. 4, 603616.

Itano, M. S., Neumann, A. K., Liu, P., Zhang, F., Gratton, E., Parak, W. J., Thompson, N. L. and Jacobson, K. (2011). DC-SIGN and influenza hemagglutinin dynamics in plasma membrane microdomains are markedly different. Biophys. J. 100, 2662-2670.

Itano, M. S., Steinhauer, C., Schmied, J. J., Forthmann, C., Liu, P., Neumann, A. K., Thompson, N. L., Tinnefeld, P. and Jacobson, K. (2012). Super-resolution imaging of C-type lectin and influenza hemagglutinin nanodomains on plasma membranes using blink microscopy. Biophys. J. 102, 1534-1542.

Jacobson, K., Mouritsen, O. G. and Anderson, R. G. (2007). Lipid rafts: at a crossroad between cell biology and physics. Nat. Cell Biol, 9, 7-14.

James, J. R., White, S. S., Clarke, R. W., Johansen, A. M., Dunne, P. D., Sleep, D. L., Fitzgerald, W. J., Davis, S. J. and Klenerman, D. (2007). Singlemolecule level analysis of the subunit composition of the T cell receptor on live $T$ cells. Proc. Natl. Acad. Sci. USA 104, 17662-17667.

Jaqaman, K., Kuwata, H., Touret, N., Collins, R., Trimble, W. S., Danuser, G. and Grinstein, S. (2011). Cytoskeletal control of CD36 diffusion promotes its receptor and signaling function. Cell 146, 593-606.

Klotzsch, E. and Schütz, G. J. (2013). A critical survey of methods to detect plasma membrane rafts. Philos. Trans. R. Soc. B 368, 20120033.

Kumar, R., Ferez, M., Swamy, M., Arechaga, I., Rejas, M. T., Valpuesta, J. M., Schamel, W. W. A., Alarcón, B. and van Santen, H. M. (2011). Increased sensitivity of antigen-experienced T cells through the enrichment of oligomeric $T$ cell receptor complexes. Immunity 35, 375-387.

Kusumi, A., Nakada, C., Ritchie, K., Murase, K., Suzuki, K., Murakoshi, H., Kasai, R. S., Kondo, J. and Fujiwara, T. (2005). Paradigm shift of the plasma membrane concept from the two-dimensional continuum fluid to the partitioned fluid: high-speed single-molecule tracking of membrane molecules. Annu. Rev. Biophys. Biomol. Struct. 34, 351-378.

Kusumi, A., Suzuki, K. G., Kasai, R. S., Ritchie, K. and Fujiwara, T. K. (2011). Hierarchical mesoscale domain organization of the plasma membrane. Trends Biochem. Sci. 36, 604-615.

Lagerholm, B. C. and Thompson, N. L. (1998). Theory for ligand rebinding at cell membrane surfaces. Biophys. J. 74, 1215-1228.

Lajoie, P., Goetz, J. G., Dennis, J. W. and Nabi, I. R. (2009). Lattices, rafts, and scaffolds: domain regulation of receptor signaling at the plasma membrane. J. Cell Biol. 185, 381-385.

Larghi, P., Williamson, D. J., Carpier, J.-M., Dogniaux, S., Chemin, K., Bohineust, A., Danglot, L., Gaus, K., Galli, T. and Hivroz, C. (2013). VAMP7 controls $\mathrm{T}$ cell activation by regulating the recruitment and phosphorylation of vesicular Lat at TCR-activation sites. Nat. Immunol. 14, 723-731.

Lillemeier, B. F., Mörtelmaier, M. A., Forstner, M. B., Huppa, J. B., Groves, J. T. and Davis, M. M. (2010). TCR and Lat are expressed on separate protein islands on T cell membranes and concatenate during activation. Nat. Immunol. 11, 90-96.

Lin, W.-C., Iversen, L., Tu, H.-L., Rhodes, C., Christensen, S. M., Iwig, J. S., Hansen, S. D., Huang, W. Y. C. and Groves, J. T. (2014). H-Ras forms dimers on membrane surfaces via a protein-protein interface. Proc. Natl. Acad. Sci. USA 111, 2996-3001.

Lingwood, D. and Simons, K. (2010). Lipid rafts as a membrane-organizing principle. Science 327, 46-50.

Liu, P., Wang, X., Itano, M. S., Neumann, A. K., Jacobson, K. and Thompson, N. L. (2012). The formation and stability of DC-SIGN microdomains require its extracellular moiety. Traffic 13, 715-726.

Liu, P., Wang, X., Itano, M. S., Neumann, A. K., de Silva, A. M., Jacobson, K. and Thompson, N. L. (2014). Low copy numbers of DC-SIGN in cell membrane microdomains: implications for structure and function. Traffic 15, 179-196.

Luo, T., Mohan, K., Iglesias, P. A. and Robinson, D. N. (2013). Molecular mechanisms of cellular mechanosensing. Nat. Mater. 12, 1064-1071.

Lynch, M. (2013). Evolutionary diversification of the multimeric states of proteins. Proc. Natl. Acad. Sci. USA 110, E2821-E2828.

Manzo, C., Torreno-Pina, J. A., Joosten, B., Reinieren-Beeren, I., Gualda, E. J., Loza-Alvarez, P., Figdor, C. G., Garcia-Parajo, M. F. and Cambi, A. (2012). The neck region of the C-type lectin DC-SIGN regulates its surface spatiotemporal organization and virus-binding capacity on antigen-presenting cells. J. Biol. Chem. 287, 38946-38955.

Mattila, P. K., Feest, C., Depoil, D., Treanor, B., Montaner, B., Otipoby, K. L., Carter, R., Justement, L. B., Bruckbauer, A. and Batista, F. D. (2013). The actin and tetraspanin networks organize receptor nanoclusters to regulate $B$ cell receptor-mediated signaling. Immunity $38,461-474$.

Maxfield, F. R. (2002). Plasma membrane microdomains. Curr. Opin. Cell Biol. 14, 483-487.

Mayor, S. and Rao, M. (2004). Rafts: scale-dependent, active lipid organization at the cell surface. Traffic 5, 231-240. 
Mayor, S. and Riezman, H. (2004). Sorting GPI-anchored proteins. Nat. Rev. Mol. Cell Biol. 5, 110-120.

Molnár, E., Swamy, M., Holzer, M., Beck-García, K., Worch, R., Thiele, C., Guigas, G., Boye, K., Luescher, I. F., Schwille, P. et al. (2012). Cholesterol and sphingomyelin drive ligand-independent T-cell antigen receptor nanoclustering. J. Biol. Chem. 287, 42664-42674.

Murakoshi, H., lino, R., Kobayashi, T., Fujiwara, T., Ohshima, C., Yoshimura, A. and Kusumi, A. (2004). Single-molecule imaging analysis of Ras activation in living cells. Proc. Natl. Acad. Sci. USA 101, 7317-7322.

Nicolson, G. L. (2013). The Fluid-Mosaic Model of Membrane Structure: still relevant to understanding the structure, function and dynamics of biological membranes after more than 40 years. Biochim. Biophys. Acta. 1838, 1451-1466.

Parton, R. G. and Hancock, J. F. (2004). Lipid rafts and plasma membrane microorganization: insights from Ras. Trends Cell Biol. 14, 141-147.

Pike, L. J. (2006). Rafts defined: a report on the Keystone Symposium on Lipid Rafts and Cell Function. J. Lipid Res. 47, 1597-1598.

Plowman, S. J., Muncke, C., Parton, R. G. and Hancock, J. F. (2005). H-ras, Kras, and inner plasma membrane raft proteins operate in nanoclusters with differential dependence on the actin cytoskeleton. Proc. Natl. Acad. Sci. USA 102, 15500-15505.

Prior, I. A., Muncke, C., Parton, R. G. and Hancock, J. F. (2003). Direct visualization of Ras proteins in spatially distinct cell surface microdomains. J. Cell Biol. 160, 165-170.

Rossier, O., Octeau, V., Sibarita, J.-B., Leduc, C., Tessier, B., Nair, D. Gatterdam, V., Destaing, O., Albigès-Rizo, C., Tampé, R. et al. (2012). Integrins $\beta 1$ and $\beta 3$ exhibit distinct dynamic nanoscale organizations inside focal adhesions. Nat. Cell Biol. 14, 1057-1067.

Schamel, W. W. and Alarcón, B. (2013). Organization of the resting TCR in nanoscale oligomers. Immunol. Rev. 251, 13-20.

Schamel, W. W. A., Arechaga, I., Risueño, R. M., van Santen, H. M., Cabezas, P., Risco, C., Valpuesta, J. M. and Alarcón, B. (2005). Coexistence of multivalent and monovalent TCRs explains high sensitivity and wide range of response. J. Exp. Med. 202, 493-503.

Sengupta, P., Jovanovic-Talisman, T., Skoko, D., Renz, M., Veatch, S. L. and Lippincott-Schwartz, J. (2011). Probing protein heterogeneity in the plasma membrane using PALM and pair correlation analysis. Nat. Methods 8, 969-975.

Serrano-Gómez, D., Sierra-Filardi, E., Martínez-Nuñez, R. T., Caparrós, E., Delgado, R., Muñoz-Fernández, M. A., Abad, M. A., Jimenez-Barbero, J., Leal, M. and Corbí, A. L. (2008). Structural requirements for multimerization of the pathogen receptor dendritic cell-specific ICAM3-grabbing non-integrin (CD209) on the cell surface. J. Biol. Chem. 283, 3889-3903.

Sharma, P., Varma, R., Sarasij, R. C., Ira, Gousset, K., Krishnamoorthy, G., Rao, M. and Mayor, S. (2004). Nanoscale organization of multiple GPIanchored proteins in living cell membranes. Cell 116, 577-589.

Sheetz, M. P. (1983). Membrane skeletal dynamics: role in modulation of red cell deformability, mobility of transmembrane proteins, and shape. Semin. Hematol. 20, 175-188.

Sherman, E., Barr, V., Manley, S., Patterson, G., Balagopalan, L., Akpan, I., Regan, C. K., Merrill, R. K., Sommers, C. L., Lippincott-Schwartz, J. et al. (2011). Functional nanoscale organization of signaling molecules downstream of the T cell antigen receptor. Immunity 35, 705-720.

Simons, K. and Toomre, D. (2000). Lipid rafts and signal transduction. Nat. Rev Mol. Cell Biol. 1, 31-39.

Singer, S. J. (1977). Thermodynamics, the structure of integral membrane proteins, and transport. J. Supramol. Struct. 6, 313-323.
Singer, S. J. and Nicolson, G. L. (1972). The fluid mosaic model of the structure of cell membranes. Science 175, 720-731.

Suzuki, K. G. N., Fujiwara, T. K., Sanematsu, F., lino, R., Edidin, M. and Kusumi, A. (2007). GPI-anchored receptor clusters transiently recruit Lyn and $\mathrm{G}$ alpha for temporary cluster immobilization and Lyn activation: single-molecule tracking study 1. J. Cell Biol. 177, 717-730.

Suzuki, K. G. N., Kasai, R. S., Hirosawa, K. M., Nemoto, Y. L., Ishibashi, M., Miwa, Y., Fujiwara, T. K. and Kusumi, A. (2012). Transient GPI-anchored protein homodimers are units for raft organization and function. Nat. Chem. Biol. 8, 774-783.

Tian, T., Harding, A., Inder, K., Plowman, S., Parton, R. G. and Hancock, J. F. (2007). Plasma membrane nanoswitches generate high-fidelity Ras signal transduction. Nat. Cell Biol. 9, 905-914.

Torreno-Pina, J. A., Castro, B. M., Manzo, C., Buschow, S. I., Cambi, A. and Garcia-Parajo, M. F. (2014). Enhanced receptor-clathrin interactions induced by $\mathrm{N}$-glycan-mediated membrane micropatterning. Proc. Natl. Acad. Sci. USA 111, 11037-11042.

van der Schaar, H. M., Rust, M. J., Waarts, B.-L., van der Ende-Metselaar, H., Kuhn, R. J., Wilschut, J., Zhuang, X. and Smit, J. M. (2007). Characterization of the early events in dengue virus cell entry by biochemical assays and singlevirus tracking. J. Virol. 81, 12019-12028.

van Meer, G., Stelzer, E. H., Wijnaendts-van-Resandt, R. W. and Simons, K. (1987). Sorting of sphingolipids in epithelial (Madin-Darby canine kidney) cells. J. Cell Biol. 105, 1623-1635.

van Zanten, T. S., Cambi, A., Koopman, M., Joosten, B., Figdor, C. G. and Garcia-Parajo, M. F. (2009). Hotspots of GPI-anchored proteins and integrin nanoclusters function as nucleation sites for cell adhesion. Proc. Natl. Acad. Sci. USA 106, 18557-18562.

Varma, R. and Mayor, S. (1998). GPI-anchored proteins are organized in submicron domains at the cell surface. Nature 394, 798-801.

Vasta, G. R. (2009). Roles of galectins in infection. Nat. Rev. Microbiol. 7, 424-438.

Veatch, S. L., Chiang, E. N., Sengupta, P., Holowka, D. A. and Baird, B. A. (2012). Quantitative nanoscale analysis of IgE-FceRI clustering and coupling to early signaling proteins. J. Phys. Chem. B 116, 6923-6935.

Williamson, D. J., Owen, D. M., Rossy, J., Magenau, A., Wehrmann, M., Gooding, J. J. and Gaus, K. (2011). Pre-existing clusters of the adaptor Lat do not participate in early $\mathrm{T}$ cell signaling events. Nat. Immunol. 12, 655662 .

Willig, K. I. and Barrantes, F. J. (2014). Recent applications of superresolution microscopy in neurobiology. Curr. Opin. Chem. Biol. 20, 16-21.

Wilson, B. S., Pfeiffer, J. R., Surviladze, Z., Gaudet, E. A. and Oliver, J. M. (2001). High resolution mapping of mast cell membranes reveals primary and secondary domains of Fc(epsilon)RI and LAT. J. Cell Biol. 154, 645-658.

Xu, K., Zhong, G. and Zhuang, X. (2013). Actin, spectrin, and associated proteins form a periodic cytoskeletal structure in axons. Science 339, 452-456.

Yáñez-Mó, M., Barreiro, O., Gordon-Alonso, M., Sala-Valdés, M. and SánchezMadrid, F. (2009). Tetraspanin-enriched microdomains: a functional unit in cell plasma membranes. Trends Cell Biol. 19, 434-446.

Yang, J. and Reth, M. (2010). Oligomeric organization of the B-cell antigen receptor on resting cells. Nature 467, 465-469.

Yu, Q. D., Oldring, A. P., Powlesland, A. S., Tso, C. K. W., Yang, C., Drickamer, K. and Taylor, M. E. (2009). Autonomous tetramerization domains in the glycanbinding receptors DC-SIGN and DC-SIGNR. J. Mol. Biol. 387, 1075-1080.

Yu, C. H., Luo, W. and Sheetz, M. P. (2012). Spatial-temporal reorganization of activated integrins. Cell Adh. Migr. 6, 280-284. 